\title{
Charge correlations and their photoinduced dynamics in charge-ordered organic ferroelectrics
}

\author{
Hirotake Itoh $\odot,{ }^{1,}{ }^{*}$ Hirokazu Obatake, ${ }^{1}$ Rina Fujiwara, ${ }^{1}$ Yohei Kawakami $\odot,{ }^{1}$ Kaoru Yamamoto $\odot,{ }^{2}$ \\ Martin Dressel $\odot,^{3}$ and Shinichiro Iwai ${ }^{1, \dagger}$ \\ ${ }^{1}$ Department of Physics, Tohoku University, Sendai 980-8578, Japan \\ ${ }^{2}$ Department of Applied Physics, Okayama University of Science, Okayama 700-0005, Japan \\ ${ }^{3}$ 1. Physikalisches Institut, Universität Stuttgart, 70569 Stuttgart, Germany
}

(Received 22 October 2020; revised 10 May 2021; accepted 8 July 2021; published 13 August 2021)

\begin{abstract}
By employing THz absorption and emission spectroscopy we were able to disentangle long-range charge order with a hundreds micrometer scale and short-range charge fluctuations that occur well above the charge-order phase transition in quasi-one-dimensional organic ferroelectrics, $(\mathrm{TMTTF})_{2} X\left(X=\mathrm{AsF}_{6}, \mathrm{PF}_{6}\right.$, and $\left.\mathrm{SbF}_{6}\right)$. While long-range charge order melts by photoexcitation irrespective of temperature or chemical pressure, short-range fluctuations are actually enhanced close to the charge-order phase boundary for $X=\mathrm{AsF}_{6}$ and $\mathrm{PF}_{6}$. Our findings reveal that short-range fluctuations show various photoresponses depending on both temperature and electronic parameters, providing a design strategy for nonequilibrium states.
\end{abstract}

DOI: 10.1103/PhysRevResearch.3.L032043

Short-range electronic correlations have been the key issue in condensed-matter physics for decades [1,2]. They often spontaneously emerge as spatially inhomogeneous domains with nanometer-scale structures around boundaries between competing electronic phases in strongly correlated systems $[3,4]$, triggering exotic aspects of high-temperature superconductivity [5] or colossal magnetoresistance [6]. Sometimes the short-range correlations themselves govern the electronic state of solids in the absence of long-range order, showing unique properties such as relaxor ferroelectricity, spin/dipole liquid, or charge glass, which are presently the subjects of intensive discussions [7-11].

Particularly intriguing is the role in photoinduced phase transitions [12-14]. The short-range correlations or fluctuations not only govern their ultrafast dynamics $[15,16]$, but also become precursors from where macroscopic metallic states grow, when introduced into a charge-order (CO) background [17-19]. Moreover, a photoinduced enhancement of them was recently found to be feasible exploiting the instability around the phase boundary [20] or the strong light-field effect [21,22]. Such versatile short-range dynamics offers a promising pathway to tailor nonequilibrium photoinduced states which might be thermodynamically inaccessible [23-26]. To this end timeresolved experiments are now actively conducted to directly uncover microscopic dynamics [27-30].

Quarter-filled organic conductors (TMTTF) ${ }_{2} X$ (TMTTF: tetramethyl-tetrathiafulvalene; $X$ : anion) [Fig. 1(a)] offer an

\footnotetext{
*hiroitoh@tohoku.ac.jp

†s-iwai@tohoku.ac.jp

Published by the American Physical Society under the terms of the Creative Commons Attribution 4.0 International license. Further distribution of this work must maintain attribution to the author(s) and the published article's title, journal citation, and DOI.
}

ideal platform for the research for their competing nature of electronic phases [31,32]. The dynamics of correlated charges can be studied as a function of both temperature and chemical substitution of $X$, which can tune electronic parameters via a chemical pressure effect. Therein electronic ferroelectricity is peculiarly induced by macroscopic $\mathrm{CO}$ below the transition temperature $T_{\mathrm{CO}}[33-36]$.

In this Letter, we report the charge dynamics in (TMTTF $)_{2} X$ investigated by terahertz (THz) spectroscopy. Short- and long-range charge correlations were distinctively observed via $\mathrm{THz}$ absorption and emission measurements, respectively [Fig. 1(b)]; intermolecular vibration and macroscopic polarization were measured. The short-range correlations, which survive even above $T_{\mathrm{CO}}$, exhibit a photoinduced enhancement close to the $\mathrm{CO}$ phase boundary for $X=\mathrm{AsF}_{6}$ and $\mathrm{PF}_{6}$; its amount depends on temperature. In contrast, the long-range $\mathrm{CO}$ shows a photoinduced suppression irrespective of temperature or $X$. Distinct responses indicate that charge fluctuations play the key role in photoinduced charge dynamics.

Hereafter we abbreviate TMTTF as TM. TM $\mathrm{TbF}_{6}$, $\mathrm{TM}_{2} \mathrm{AsF}_{6}$, and $\mathrm{TM}_{2} \mathrm{PF}_{6}$ were synthesized by the electrochemical method [31,37]. Bulk single crystals with triclinic symmetry [38-41] having typical sizes of $2 \times 1 \times 0.1 \mathrm{~mm}^{3}$ ( $a b$ plane) were mounted on copper plates and placed in an He exchange gas cryostat (Oxford Optistat-CF). As shown in Fig. 1(a), TM dimers stack along the $a$ axis (conducting axis). Below $T_{\mathrm{CO}}$, each dimer undergoes charge disproportionation due to a strong Coulomb repulsion to have $(\mathrm{TM})^{+0.5+\delta}$ and $(\mathrm{TM})^{+0.5-\delta}(2 \delta \sim 0.2$ [32] $)$. Consequent local electric dipoles form long-range order or macroscopic $\boldsymbol{P}$ (electronic ferroelectricity), which will be inverted upon interchanging charge-rich/charge-poor sites.

We performed two kinds of $\mathrm{THz}$ measurements as shown in Fig. 1(b): THz absorption (transmission), and $\mathrm{THz}$ emission triggered by $1.55-\mathrm{eV}$ femtosecond pulses, reflecting 


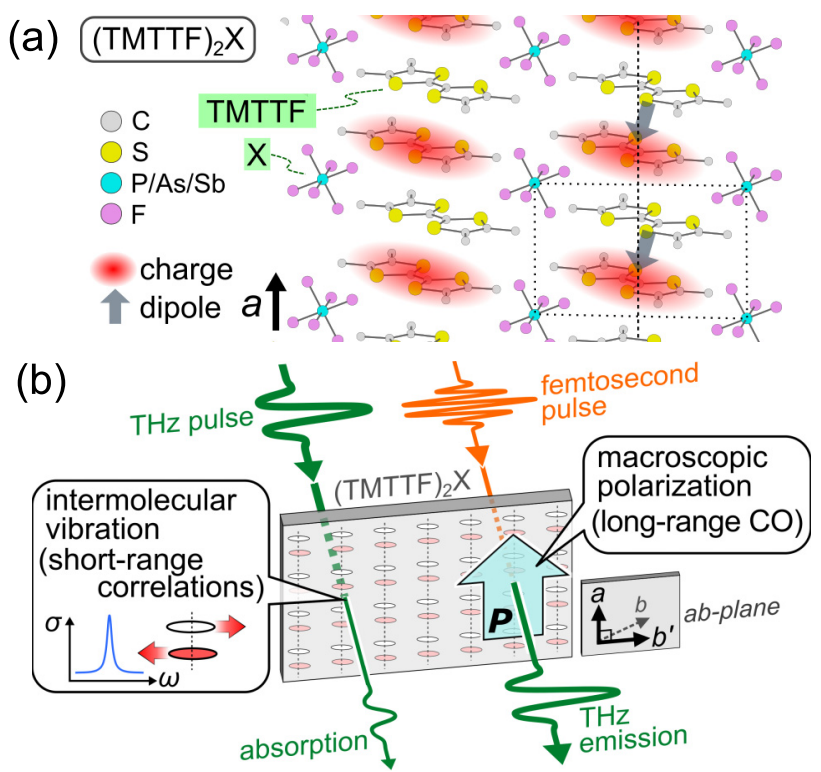

FIG. 1. (a) Schematic of $\mathrm{TM}_{2} X$ crystal (data taken from Ref. [42]), with $\mathrm{CO}$ and an expected electric dipole (gray arrows). The projected unit cell and stacking direction of TMTTF molecules $(\| a)$ is indicated by dotted and dashed lines, respectively. (b) Schematics of THz absorption (left) and THz emission (right) experiments. The THz polarization $\left(\| b^{\prime}\right)$ is perpendicular to the stack; $b^{\prime}$ denotes the projection of the $b$ axis perpendicular to the $a$ axis, in the $a b$ plane.

intradimer charge disproportionation and macroscopic polarization, respectively, allowing us to selectively discuss shortand long-range charge correlations. The polarization of the light was parallel to the $b^{\prime}$ axis (perpendicular to the stack [Fig. 1(b)]). The time-domain waveform of the THz electric field was collected by electro-optic sampling (time-domain spectroscopy). The experimental details are given in the Supplemental Material [43].

Figure 2(a) shows the steady-state absorption (optical density, OD) spectra of $\mathrm{TM}_{2} \mathrm{AsF}_{6}$; the same data are shown in the waterfall and image plot. Here, we concentrate on a peak at $\sim 66 \mathrm{~cm}^{-1}$ which developed with decreasing temperature down to below $T_{\mathrm{CO}}=102 \mathrm{~K}$. This mode has been assigned to a vibration between a pair of TM molecules having an antiphase translation along the longitudinal direction $\left(T_{c}\right.$ mode [32]), which becomes infrared active by charge disproportionation yielding intradimer electric dipoles [Fig. 1(a)]. Hence its oscillator strength is a measure of how short-range charge correlations develop, as will be shown later. Based on this assignment, we will refer to this peak as the SR (short-range) peak.

Figure 2(b) shows optical conductivity spectra for all compounds. Again, the SR peak of $\mathrm{TM}_{2} \mathrm{AsF}_{6}$ at $\sim 66 \mathrm{~cm}^{-1}$ (triangle) develops with decreasing temperature from above $T_{\mathrm{CO}}$. At a low temperature $T=20 \mathrm{~K}$, other peaks are also seen at 54,75 , and $85 \mathrm{~cm}^{-1}$, in agreement with the previous study [32], where 54- and $85-\mathrm{cm}^{-1}$ peaks represent $T_{a}$ and $T_{b}$ mode
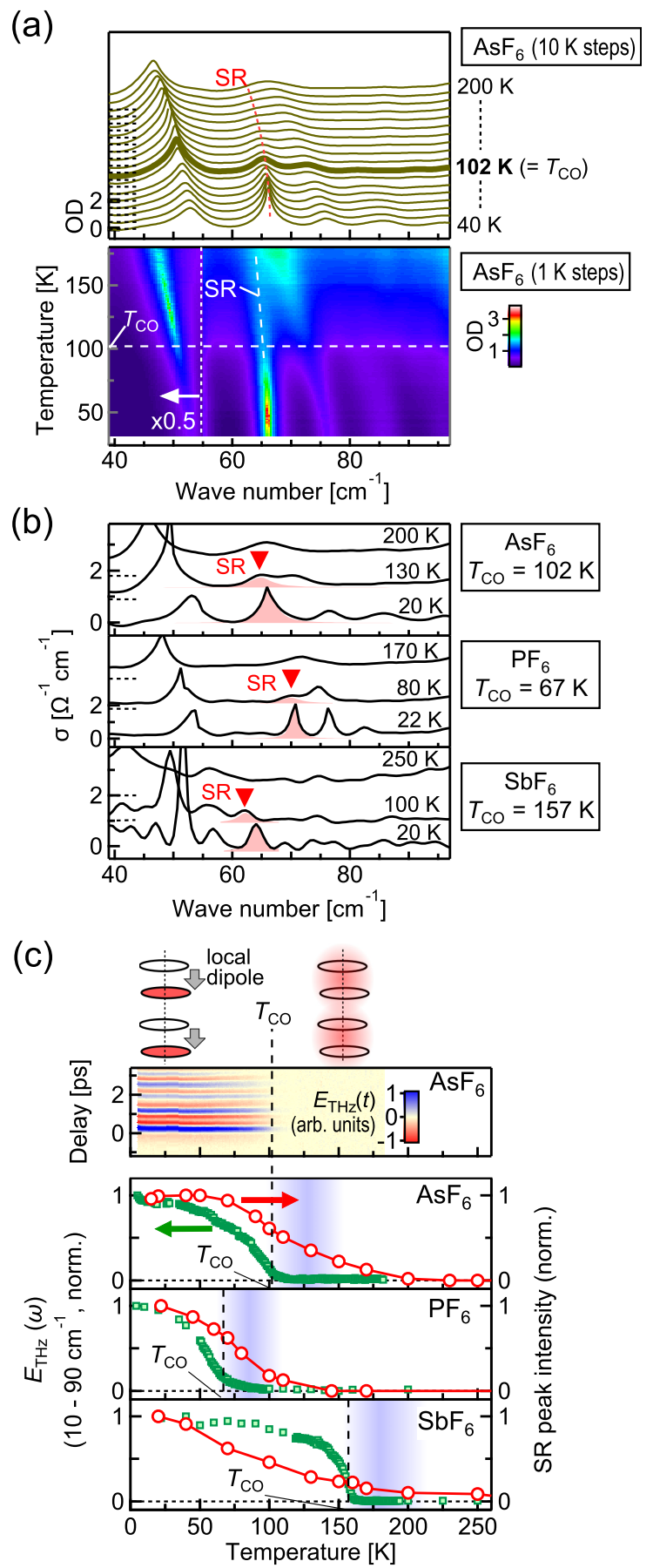

FIG. 2. (a) Absorption spectra of $\mathrm{TM}_{2} \mathrm{AsF}_{6}$. The same data, measured in $1-\mathrm{K}$ steps, are shown every $10 \mathrm{~K}$ (except for $T_{\mathrm{CO}}$ ) in the upper panel, whereas they are shown in the image plot without interpolation in the lower panel. (b) Optical conductivity spectra of $\mathrm{TM}_{2} \mathrm{AsF}_{6}$, $\mathrm{TM}_{2} \mathrm{PF}_{6}$, and $\mathrm{TM}_{2} \mathrm{SbF}_{6}$. Triangles and shaded regions indicate the SR peaks and their Lorentzian fits, respectively. Spectra at higher temperatures are vertically offset for clarity (dashed lines indicate their zeros). (c) Temperature dependences. Top panel: Time-domain waveforms of $\mathrm{THz}$ electric field $E_{\mathrm{THz}}$ emitted from $\mathrm{TM}_{2} \mathrm{AsF}_{6}$. Lower panels: Spectral intensities of $E_{\mathrm{THz}}$ and $\mathrm{SR}$ peak intensities, for $\mathrm{TM}_{2} \mathrm{AsF}_{6}, \mathrm{TM}_{2} \mathrm{PF}_{6}$, and $\mathrm{TM}_{2} \mathrm{SbF}_{6}$. 
intermolecular translations, respectively. Among them, the SR peak is the most useful probe of $\mathrm{CO}$ for its largest change against temperature. Similarly, for the analogous compound $\mathrm{TM}_{2} \mathrm{PF}_{6}$, peaks were observed in the vicinity at $22 \mathrm{~K} \ll T_{\mathrm{CO}}$; the $70-\mathrm{cm}^{-1}$ peak should be assigned to the $T_{c}$ mode or the SR peak, for its largest increase across $T_{\mathrm{CO}}$ as the SR peak of $\mathrm{TM}_{2} \mathrm{AsF}_{6}$. Given that the peak redshifts with increasing anion size, a lower frequency is expected for $\mathrm{TM}_{2} \mathrm{SbF}_{6}$ with the larger size. Accordingly, the intensity of the $64-\mathrm{cm}^{-1}$ peak $\left(20 \mathrm{~K} \ll T_{\mathrm{CO}}\right)$ shows the largest increase against temperature, hence it is reasonably assigned to the SR peak. The systematic shift is explicitly shown in Fig. S1 [43].

It is reasonable that the SR peak does not soften around $T_{\mathrm{CO}}$. In a displacive-type ferroelectrics, a soft mode is an optical phonon whose motion modulates potential $\boldsymbol{P}$. In contrast, the intermolecular vibration causing the SR peak is not infrared active until the charge disproportionation sets in around $T_{\mathrm{CO}}$; hence it cannot be a soft mode, despite its sensitivity to $\boldsymbol{P}$.

It should be noted that the SR peaks are evident even slightly above $T_{\mathrm{CO}}\left(130 \mathrm{~K}\right.$ for $\mathrm{TM}_{2} \mathrm{AsF}_{6}$ and $80 \mathrm{~K}$ for $\mathrm{TM}_{2} \mathrm{PF}_{6}$ ); the continuous change from $T \ll T_{\mathrm{CO}}$ is confirmed by absorption spectra measured in 1-K steps [Fig. 2(a)]. This is consistent with the fact that short-range correlations in general start to develop above the phase transition temperature in low dimensions [44]; intradimer charge disproportionation would randomly take place above $T_{\mathrm{CO}}$, and the fluctuations slower than the peak frequency $(\sim 1 / \mathrm{ps})$ bear their infrared activity. Such a temperature evolution of the short-range correlations is also clearly seen in the lower three panels of Fig. 2(c), where we plot the peak intensities estimated by a Lorentzian fit [shaded regions in Fig. 2(b)] to exclude contributions of adjacent peaks and background.

Although the preceding study on a one-dimensional system has pointed out a divergent increase of infrared activity near the transition [45], we could not identify it in the observed spectra [46].

Unlike diffraction experiments $[47,48]$, the SR peak alone can hardly disentangle contributions from the short- and longrange correlations. To resolve this, we also measured the $\mathrm{THz}$ emission triggered by femtosecond pulses [Fig. 1(b)]. The color map shown in the top panel of Fig. 2(c) represents the time-domain waveforms of the THz electric field $E_{\mathrm{THz}}(t)$ emitted from $\mathrm{TM}_{2} \mathrm{AsF}_{6}$. $E_{\mathrm{THz}}$ became nonzero below $T_{\mathrm{CO}}$, where $\mathrm{CO}$ accompanies macroscopic $\boldsymbol{P}$ breaking spatial inversion symmetry. Hence the emission process is attributable to optical rectification via second-order nonlinear susceptibility $\chi^{(2)}(\omega-\omega)$, which in general becomes nonzero upon losing centrosymmetry [49-51]. Accordingly the emission intensity showed the expected quadratic increase with incident light fluence, as shown in Fig. S2 [43].

Note that the $E_{\mathrm{THz}}$ signal arises from the $\mathrm{CO}$ with longrange space correlations; a $\mathrm{THz}$ wave from a bunch of nanoscaled domains having antiparallel $\boldsymbol{P}$ (antiphase charge configuration) will destructively interfere and cancel out, since inversion of $\boldsymbol{P}$ will invert the sign of $\chi^{(2)}$ or the resultant $E_{\mathrm{THz}}$. This behavior is distinct from the SR peak absorption, which is activated by intradimer charge disproportionation irrespective of its phase.

To confirm the long-range correlations, we performed THz-emission microscopy. By focusing the fundamental
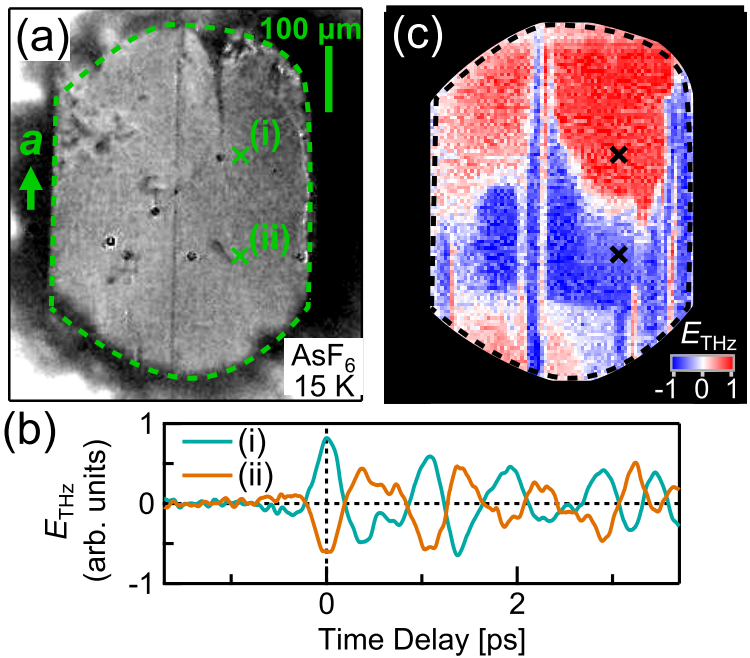

FIG. 3. (a) Reflection image of $\mathrm{TM}_{2} \mathrm{AsF}_{6}$ sample at $15 \mathrm{~K}$. (b) Time-domain $\mathrm{THz}$ waveforms observed by irradiating the positions (i) and (ii) with fundamental light having a 5- $\mu \mathrm{m}$-diameter spot focused by an objective lens (numerical aperture NA $=0.13$ ). The fluence was $5 \mathrm{~mJ} / \mathrm{cm}^{2}$. (c) Raster scan of THz emission: 5- $\mu \mathrm{m}$-step map of $E_{\mathrm{THz}}(0 \mathrm{ps})$

beam onto $\mathrm{TM}_{2} \mathrm{AsF}_{6}, \mathrm{THz}$ emission occurs from the irradiated 5- $\mu \mathrm{m}$ spot, e.g. position (i) or (ii) shown in Fig. 3(a). As shown in Fig. 3(b), the resultant $E_{\mathrm{THz}}$ exhibited a positiondependent sign reversal. This should be due to the inversion of $\chi^{(2)}$ upon inversion of the order parameter $\boldsymbol{P}$; hence the $E_{\mathrm{THz}}(0 \mathrm{ps})$ value works as a measure of the sign of $\boldsymbol{P}[52]$. As shown in Fig. 3(c), its color map, or raster scan of the $\mathrm{THz}$ emission, clearly evidences antiparallel $\boldsymbol{P}$ domains with correlations as long as hundreds of microns in the $a b$ plane. Such large domains can avoid the cancellation effect, and in fact, the $E_{\mathrm{THz}}$ signal was successfully observed even in nonmicroscopy measurements [Fig. 2(c)] reflecting the long-range $\mathrm{CO}$ (see Supplemental Material for a quantitative characterization [43]).

In the lower three panels of Fig. 2(c), we plot the intensities of the amplitude spectra $E_{\mathrm{THz}}(\omega)$, the Fourier transform of time-domain waveforms, for all compounds (see Figs. S2$\mathrm{S} 4$ for spectral shapes). The onsets of $E_{\mathrm{THz}}$ are almost at $T_{\mathrm{CO}}$, in contrast to those of the SR peak intensity which are $\sim 50 \mathrm{~K}$ above $T_{\mathrm{CO}}$. This unambiguously demonstrates that the short-range correlations develop above $T_{\mathrm{CO}}$ activating the SR peak, followed by the formation of the long-range $\mathrm{CO}$ triggering $\mathrm{THz}$ emission below $T_{\mathrm{CO}}$. Therefore they provide complementary insight on the charge dynamics.

The development of $\mathrm{CO}$ has been quantitatively studied by midinfrared and Raman spectroscopy via intramolecular phonon modes showing a charge-sensitive frequency shift or consequent splitting [32,53-58]. Therein no particular attention was paid to the behavior above $T_{\mathrm{CO}}$, probably because it is not easy to identify a weak satellite peak with a small shift compared to its width. In contrast, the SR peak, which is intermolecular vibration focused in this work, is not hindered by such an overlap and hence the development of its intensity could sensitively be captured [59]. 
(a)

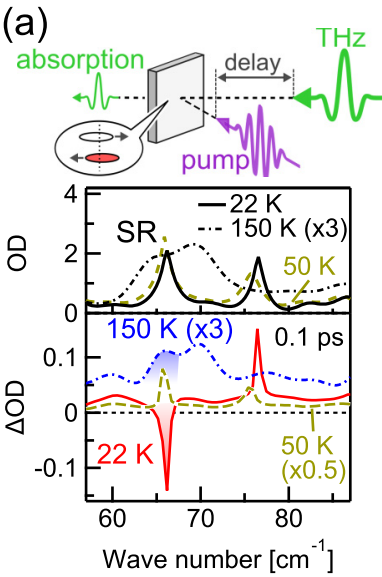

(b) $T$
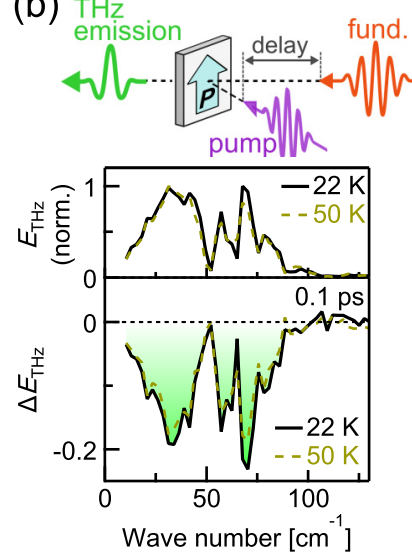

(c)

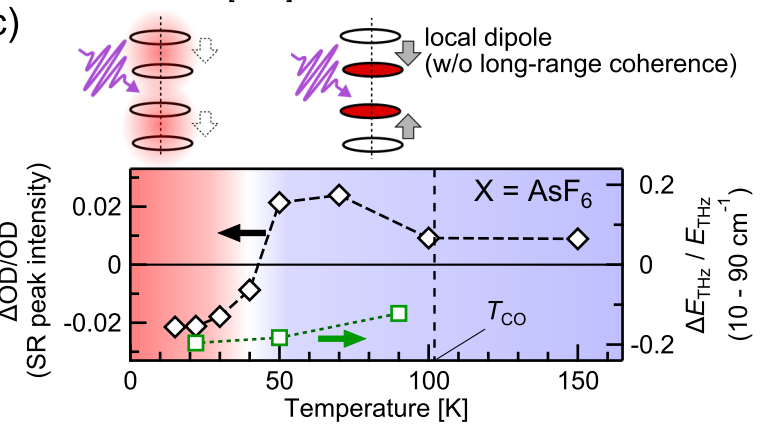

FIG. 4. (a) and (b) show the schematics of the optical-pump THz-absorption-probe and $\mathrm{THz}$-emission-probe experiment, respectively, and the results for $\mathrm{TM}_{2} \mathrm{AsF}_{6}$. The upper and lower panel show steady-state $\mathrm{THz}$ absorption (emission) spectra and their photoinduced change, respectively. OD at $150 \mathrm{~K}$ is vertically offset by -2.6 for clarity. (c) Temperature dependence of the photoinduced changes of the SR peak intensity (left axis) and $\mathrm{THz}$ emission (right axis). The schematics show the photoinduced charge dynamics therein.

Having identified the probes for short- and long-range charge correlations, we can now study their photoinduced dynamics. To that end we measured the photoinduced change of $\mathrm{THz}$ absorption and emission as schematically shown in Figs. 4(a) and 4(b), respectively, without the contribution of the pump-pulse-induced $\mathrm{THz}$ emission [60]. The 0.89-eV pump light $(\| a)$ above the $\mathrm{CO}$ gap $\Delta_{\mathrm{CO}} \sim 0.1 \mathrm{eV}$ [31] corresponds to the charge transfer excitation [61]. The pump fluence was $0.2 \mathrm{~mJ} / \mathrm{cm}^{2}$; the laser heating effect does not affect the following discussions, wherein the resultant rise of crystal temperature is as small as $\sim 5 \mathrm{~K}$ [43]. It is known that the temporal resolution of transient $\mathrm{THz}$ time-domain measurements can be as good as the pump duration, currently $0.1 \mathrm{ps}$, despite a chirped $\mathrm{THz}$ probe pulse with a duration of several picoseconds [62,63] (see Supplemental Material for details [43]).

Figure 4(a) shows steady-state absorption spectra of $\mathrm{TM}_{2} \mathrm{AsF}_{6}$ around the SR peak (upper panel), and their changes at $0.1 \mathrm{ps}$ (pump pulse duration) after photoexcitation (lower panel). At $22 \mathrm{~K}$ well below $T_{\mathrm{CO}}$, the SR peak was suppressed upon photoexcitation (red line). Since such suppression was absent in the adjacent phonon peak at $\sim 76 \mathrm{~cm}^{-1}$, the result cannot be accounted for by laser heating or by screening due to photocarriers which presumably caused broad photoabsorption of $\Delta \mathrm{OD} \sim 0.02$. Therefore the suppression should be due to a photoinduced dissolution of intradimer charge disproportionation hosting the infrared activity of the SR peak. In striking contrast, at $150 \mathrm{~K}>T_{\mathrm{CO}}$ where charges only have short-range correlations without long-range CO, the SR peak was enhanced within $0.1 \mathrm{ps}$ after photoexcitation (blue dashed-dotted line). Since the change is contrary to the suppression, it is reasonable to assign the result to a photoinduced enhancement of the short-range correlations. The electronic response should be dominant in these instantaneous changes, since the timescale is much shorter than a period of the SR mode, $\left(\sim 70 \mathrm{~cm}^{-1}\right)^{-1} \sim 0.5 \mathrm{ps}$, which is the minimum duration required to define the peak frequency. Time evolutions of $\Delta$ OD are shown in Fig. S6 [43].

Since the peak at higher frequency $\left(\sim 76 \mathrm{~cm}^{-1}\right.$ at $\left.22 \mathrm{~K}\right)$ has not yet been assigned, its photoinduced change remains unresolved at present; however, it is likely insensitive to the photoinduced charge dynamics, since it does not show a noticeable change around $T_{\mathrm{CO}}$ in the steady-state spectra [Fig. 2(a)].

Figure 4(b) shows, in the same manner as Fig. 4(a), the $E_{\mathrm{THz}}$ spectra and their changes at 0.1 ps after photoexcitation. At $T=22 \mathrm{~K}, E_{\mathrm{THz}}$ was suppressed over the whole spectral range, indicating that macroscopic $\boldsymbol{P}$ vanishes upon photoexcitation; the result is consistent with the dissolution of short-range correlations [Fig. 4(a)] which inevitably collapses long-range CO. Although such a collapse is known to accompany a photoinduced insulator-metal transition in another system $[50,64,65]$, we currently cannot judge whether or not the photoinduced state is metallic before observing the $\mathrm{THz}$ dynamics along the conducting $a$ axis.

Figure 4(c) summarizes the temperature dependences. With $T$ increasing from $15 \mathrm{~K}$, the photoinduced change of the SR peak turns from suppression to enhancement near $40 \mathrm{~K}$ below $T_{\mathrm{CO}}$, and the enhancement is also observed around and above $T_{\mathrm{CO}}$. This reveals that the photoinduced change of the short-range $\mathrm{CO}$ undergoes a crossover; the crossing temperature $\sim 40 \mathrm{~K}$ reflecting the competition between the suppression and enhancement is characteristic of the photoinduced states, and accordingly equilibrium data show no anomaly around the temperature (Fig. 2). Although $\mathrm{CO}$ in the higher-temperature side is closer to its thermal melting point at $T_{\mathrm{CO}}$, the short-range fluctuations therein are actually enhanced upon photoexcitation. Its amount tends to become smaller at higher temperature; to resolve the detail, a further study including sample dependence is required. On the other hand, $\mathrm{THz}$ emission or long-range $\mathrm{CO}$ was suppressed irrespective of temperature. Note that the signs of $\triangle \mathrm{OD}$ and $\Delta E_{\mathrm{THz}}$ are opposite above $40 \mathrm{~K}$, for example, at $50 \mathrm{~K}$ as seen in Figs. 4(a) and 4(b) (yellow dashed lines). This is explained by the difference in spatial scale; upon photoexcitation the charge distribution was randomized to have a larger intradimer disproportionation than before, while long-range $(\sim \mu \mathrm{m})$ coherence was lost, canceling macroscopic $\boldsymbol{P}$.

The amount of photoinduced change is closely related to the volume fraction of the photoexcited surface. It is estimated as $\sim 0.4 \%$ for a typical sample thickness of $100 \mu \mathrm{m}$, using a penetration depth $d \sim 0.4 \mu \mathrm{m}$ at $0.89 \mathrm{eV}$ calculated from the result of a Kramers-Kronig analysis [66]. Hence the $\sim 2 \%$ decrease of the SR peak intensity [Fig. 4(c)] suggests that 

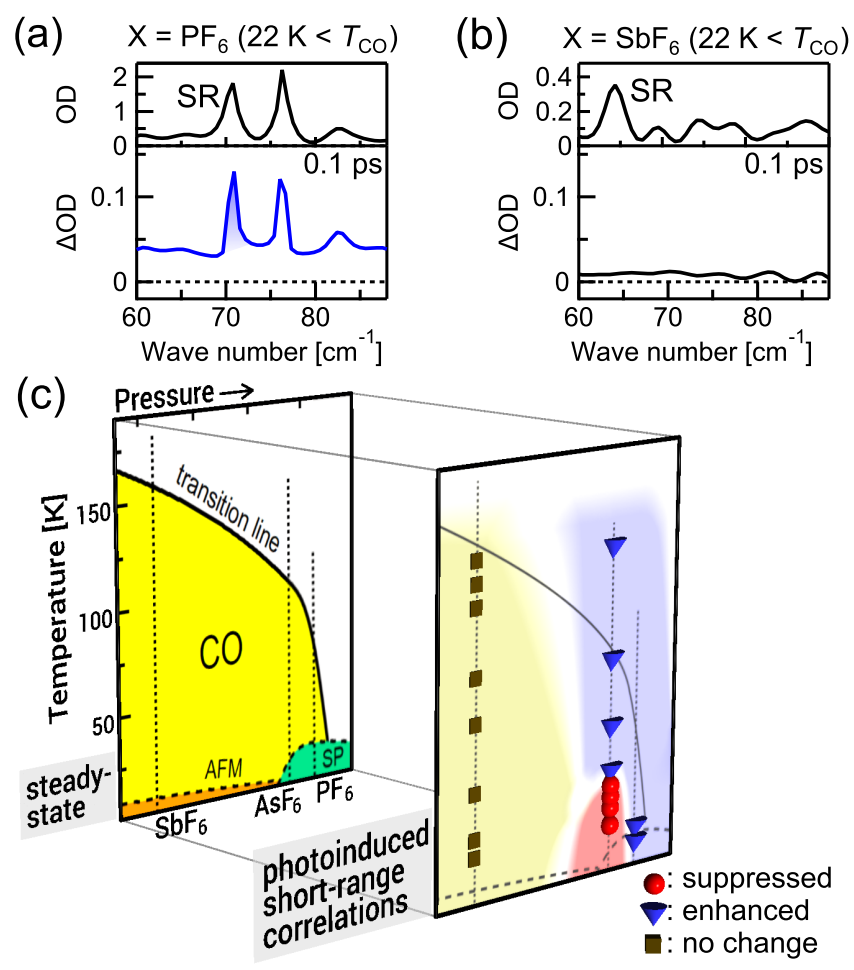

FIG. 5. (a) and (b) show results of the optical-pump THzabsorption-probe experiments for $\mathrm{TM}_{2} \mathrm{PF}_{6}$ and $\mathrm{TM}_{2} \mathrm{SbF}_{6}$, respectively, shown in the same way as Fig. 4(a). (c) Left: Steady-state temperature-pressure phase diagram of $\mathrm{TM}_{2} X$ (reproduced from Ref. [32]). AFM: antiferromagnet; SP: Spin Peierls. Right: Photoinduced change of the SR peak superimposed on the diagram. Red spheres: suppressed; blue cones: Enhanced; brown boxes: no change.

CO was almost completely dissolved. Therein the photon number was estimated as small as $\sim 0.005$ per TM molecule, implying that cooperative phenomena took place as in other photoinduced phase transition processes [17], although a fluence-dependent study is required for a detailed discussion. For $\mathrm{THz}$ emission, the volume fraction of the photoinduced area is effectively $\sim 100 \%$ because $d$ for $1.55-\mathrm{eV}$ fundamental light is comparable to that for the pump light [66], hence a larger photoinduced change is expected, as observed $(\sim 20 \%)$. A quantitative comparison of the photoinduced changes requires an analysis of domain structures, which is beyond the scope of this Letter.

To gain deeper insight into the characteristic enhancement of the short-range correlations, we performed the same experiments on other compounds. As shown in Fig. 5(a), the SR peak of $\mathrm{TM}_{2} \mathrm{PF}_{6}$ at $22 \mathrm{~K}$ was enhanced within $0.1 \mathrm{ps}$ after photoexcitation; on changing the anion from $\mathrm{AsF}_{6}$ to $\mathrm{PF}_{6}$, i.e., applying chemical pressure, the peak suppression [Fig. 4(a)] turns into the enhancement. This inversion resembles that observed for $\mathrm{TM}_{2} \mathrm{AsF}_{6}$ during heating [Fig. 4(c)], both of which correspond to approaching the $\mathrm{CO}$ transition line in the phase diagram [left panel of Fig. 5(c), reproduced from Ref. [32]]. The magnetic contribution should be absent in the inversion, since the result was invariant at a lower temperature below the spin-Peierls transition (Fig. S7 [43]). For $\mathrm{TM}_{2} \mathrm{SbF}_{6}$ located in the lower-pressure region, no change was identified in the SR peak [Figs. 5(b) and 5(c)]. THz emission of these compounds, on the other hand, was suppressed as in $\mathrm{TM}_{2} \mathrm{AsF}_{6}$ (Fig. S6 [43]), indicating that long-range $\mathrm{CO}$ is always collapsed upon photoexcitation irrespective of chemical pressure or temperature. The collapse without the SR peak suppression is attributable to the randomization of the short-range correlations [Fig. 4(c)], although the correlations in $\mathrm{TM}_{2} \mathrm{SbF}_{6}$ do not appear to be enhanced. The photoinduced change had a timescale of several picoseconds and was insensitive to temperature nor anion (Fig. S6 [43]).

In Fig. 5(c) we show on the left-hand side the steadystate temperature-pressure phase diagram reproduced from Ref. [32], while on the right-hand side we superimposed the results of the photoinduced changes of the SR peak on the diagram (original data are shown in Fig. S7 [43]). The suppression was observed only for $\mathrm{TM}_{2} \mathrm{AsF}_{6}$ at low temperatures in the $\mathrm{CO}$ phase (red spheres), where intradimer charge disproportionation is dissolved. With increasing temperature or pressure toward the $\mathrm{CO}$ phase boundary, it turned into the enhancement (blue cones). Meanwhile, when the pressure is small, no change was identified even near $T_{\mathrm{CO}}$ (brown boxes).

The various photoresponses of the short-range $\mathrm{CO}$ were found to be sensitive not only to temperature but also to pressure or the resultant electronic parameters, showing the distinctive distribution from the steady-state phase diagram. This opens up an extra route toward tailoring unprecedented nonequilibrium states, which is based on another idea rather than the suppression of competing phases as seen in the photoinduced superconductivity [67]. Although recent theoretical studies have succeeded in showing that the photoinduced modulation of short-range charge correlations is feasible [61,68,69], further investigations are indispensable to quantitatively understand the observed crossover against temperature and chemical pressure.

This work was supported by Japan Science and Technology agency (JST) CREST (JPMJCR1901), MEXT Q-LEAP (JPMXS0118067426), Japan Society for the Promotion of Science (JSPS) KAKENHI (Grants No. JP15H02100, No. JP17K14317, No. JP18H01144, No. JP20K03800, and No. JP20H05147), Hattori Hokokai Foundation, and Toyota Riken Scholar. The work at Universität Stuttgart was supported by the DFG via DR228/39-3.
[1] E. Dagotto, Complexity in strongly correlated electronic systems, Science 309, 257 (2005).

[2] M. Liu, A. J. Sternbach, and D. N. Basov, Nanoscale electrodynamics of strongly correlated quantum materials, Rep. Prog. Phys. 80, 014501 (2017).
[3] A. S. McLeod, E. van Heumen, J. G. Ramirez, S. Wang, T. Saerbeck, S. Guenon, M. Goldflam, L. Anderegg, P. Kelly, A. Mueller, M. K. Liu, I. K. Schuller, and D. N. Basov, Nanotextured phase coexistence in the correlated insulator $\mathrm{V}_{2} \mathrm{O}_{3}$, Nat. Phys. 13, 80 (2017). 
[4] A. Pustogow, A. S. McLeod, Y. Saito, D. N. Basov, and M. Dressel, Internal strain tunes electronic correlations on the nanoscale, Sci. Adv. 4, eaau9123 (2018).

[5] B. Keimer, S. A. Kivelson, M. R. Norman, S. Uchida, and J. Zaanen, From quantum matter to high-temperature superconductivity in copper oxides, Nature (London) 518, 179 (2015).

[6] Y. Tokura, Critical features of colossal magnetoresistive manganites, Rep. Prog. Phys. 69, 797 (2006).

[7] R. A. Cowley, S. N. Gvasaliya, S. G. Lushnikov, B. Roessli, and G. M. Rotaru, Relaxing with relaxors: a review of relaxor ferroelectrics, Adv. Phys. 60, 229 (2011).

[8] M. Dressel and A. Pustogow, Electrodynamics of quantum spin liquids, J. Phys.: Condens. Matter 30, 203001 (2018).

[9] N. Hassan, S. Cunningham, M. Mourigal, E. I. Zhilyaeva, S. A. Torunova, R. N. Lyubovskaya, J. A. Schlueter, and N. Drichko, Evidence for a quantum dipole liquid state in an organic quasitwo-dimensional material, Science 360, 1101 (2018).

[10] F. Kagawa, T. Sato, K. Miyagawa, K. Kanoda, Y. Tokura, K. Kobayashi, R. Kumai, and Y. Murakami, Charge-cluster glass in an organic conductor, Nat. Phys. 9, 419 (2013).

[11] S. Sasaki, K. Hashimoto, R. Kobayashi, K. Itoh, S. Iguchi, Y. Nishio, Y. Ikemoto, T. Moriwaki, N. Yoneyama, M. Watanabe, A. Ueda, H. Mori, K. Kobayashi, R. Kumai, Y. Murakami, J. Müller, and T. Sasaki, Crystallization and vitrification of electrons in a glass-forming charge liquid, Science 357, 1381 (2017).

[12] K. Nasu, Itinerant type many-body theories for photo-induced structural phase transitions, Rep. Prog. Phys. 67, 1607 (2004).

[13] M. Kuwata-Gonokami and S. Koshihara (eds.), Special issue on photo-induced phase transitions and their dynamics, J. Phys. Soc. Jpn. 75, issue 1 (2006).

[14] C. Giannetti, M. Capone, D. Fausti, M. Fabrizio, F. Parmigiani, and D. Mihailovic, Ultrafast optical spectroscopy of strongly correlated materials and high-temperature superconductors: A non-equilibrium approach, Adv. Phys. 65, 58 (2016).

[15] M. C. Langner, S. Zhou, G. Coslovich, Y.-D. Chuang, Y. Zhu, J. S. Robinson, W. F. Schlotter, J. J. Turner, M. P. Minitti, R. G. Moore, W. S. Lee, D. H. Lu, D. Doering, P. Denes, Y. Tomioka, Y. Tokura, R. A. Kaindl, and R. W. Schoenlein, Ultrafast Xray and optical signatures of phase competition and separation underlying the photoinduced metallic phase in $\operatorname{Pr}_{1-x} \mathrm{Ca}_{x} \mathrm{MnO}_{3}$, Phys. Rev. B 92, 155148 (2015).

[16] S. D. Conte, L. Vidmar, D. Golež, M. Mierzejewski, G. Soavi, S. Peli, F. Banfi, G. Ferrini, R. Comin, B. M. Ludbrook, L. Chauviere, N. D. Zhigadlo, H. Eisaki, M. Greven, S. Lupi, A. Damascelli, D. Brida, M. Capone, J. Bonča, G. Cerullo et al., Snapshots of the retarded interaction of charge carriers with ultrafast fluctuations in cuprates, Nat. Phys. 11, 421 (2015).

[17] S. Iwai, K. Yamamoto, A. Kashiwazaki, F. Hiramatsu, H. Nakaya, Y. Kawakami, K. Yakushi, H. Okamoto, H. Mori, and Y. Nishio, Photoinduced Melting of a Stripe-Type Charge-Order and Metallic Domain Formation in a Layered BEDT-TTFBased Organic Salt, Phys. Rev. Lett. 98, 097402 (2007).

[18] H. Nakaya, K. Itoh, Y. Takahashi, H. Itoh, S. Iwai, S. Saito, K. Yamamoto, and K. Yakushi, Terahertz responses of the hightemperature metallic phase and photoinduced metallic state in the ferroelectric charge-ordered organic salt $\alpha-(\mathrm{ET})_{2} \mathrm{I}_{3}$, Phys. Rev. B 81, 155111 (2010).

[19] Y. Kawakami, T. Fukatsu, Y. Sakurai, H. Unno, H. Itoh, S. Iwai, T. Sasaki, K. Yamamoto, K. Yakushi, and K. Yonemitsu,
Early-Stage Dynamics of Light-Matter Interaction Leading to the Insulator-to-Metal Transition in a Charge Ordered Organic Crystal, Phys. Rev. Lett. 105, 246402 (2010).

[20] Y. Wang, C.-C. Chen, B. Moritz, and T. P. Devereaux, LightEnhanced Spin Fluctuations and d-Wave Superconductivity at a Phase Boundary, Phys. Rev. Lett. 120, 246402 (2018).

[21] T. Ishikawa, Y. Sagae, Y. Naitoh, Y. Kawakami, H. Itoh, K. Yamamoto, K. Yakushi, H. Kishida, T. Sasaki, S. Ishihara, Y. Tanaka, K. Yonemitsu, and S. Iwai, Optical freezing of charge motion in an organic conductor, Nat. Commun. 5, 5528 (2014).

[22] Y. Naitoh, Y. Kawakami, T. Ishikawa, Y. Sagae, H. Itoh, K. Yamamoto, T. Sasaki, M. Dressel, S. Ishihara, Y. Tanaka, K. Yonemitsu, and S. Iwai, Ultrafast response of plasmalike reflectivity edge in (TMTTF $)_{2} \mathrm{AsF}_{6}$ driven by a 7 -fs 1.5 -cycle strong-light field, Phys. Rev. B 93, 165126 (2016).

[23] H. Ichikawa, S. Nozawa, T. Sato, A. Tomita, K. Ichiyanagi, M. Chollet, L. Guerin, N. Dean, A. Cavalleri, S. Adachi, T. Arima, H. Sawa, Y. Ogimoto, M. Nakamura, R. Tamaki, K. Miyano, and S. Koshihara, Transient photoinduced "hidden" phase in a manganite, Nat. Mater. 10, 101 (2011).

[24] T.-R. T. Han, F. Zhou, C. D. Malliakas, P. M. Duxbury, S. D. Mahanti, M. G. Kanatzidis, and C.-Y. Ruan, Exploration of metastability and hidden phases in correlated electron crystals visualized by femtosecond optical doping and electron crystallography, Sci. Adv. 1, e1400173 (2015).

[25] D. Fausti, R. I. Tobey, N. Dean, S. Kaiser, A. Dienst, M. C. Hoffmann, S. Pyon, T. Takayama, H. Takagi, and A. Cavalleri, Light-induced superconductivity in a stripe-ordered cuprate, Science 331, 189 (2011).

[26] Z. Sun and A. J. Millis, Transient Trapping into Metastable States in Systems with Competing Orders, Phys. Rev. X 10, 021028 (2020).

[27] S. A. Dönges, O. Khatib, B. T. O’Callahan, J. M. Atkin, J. H. Park, D. Cobden, and M. B. Raschke, Ultrafast nanoimaging of the photoinduced phase transition dynamics in $\mathrm{VO}_{2}$, Nano Lett. 16, 3029 (2016).

[28] A. Zong, A. Kogar, Y.-Q. Bie, T. Rohwer, C. Lee, E. Baldini, E. Ergeçen, M. B. Yilmaz, B. Freelon, E. J. Sie, H. Zhou, J. Straquadine, P. Walmsley, P. E. Dolgirev, A. V. Rozhkov, I. R. Fisher, P. Jarillo-Herrero, B. V. Fine, and N. Gedik, Evidence for topological defects in a photoinduced phase transition, Nat. Phys. 15, 27 (2019).

[29] Y. A. Gerasimenko, P. Karpov, I. Vaskivskyi, S. Brazovskii, and D. Mihailovic, Intertwined chiral charge orders and topological stabilization of the light-induced state of a prototypical transition metal dichalcogenide, npj Quantum Mater. 4, 32 (2019).

[30] T. Ishikawa, S. A. Hayes, S. Keskin, G. Corthey, M. Hada, K. Pichugin, A. Marx, J. Hirscht, K. Shionuma, K. Onda, Y. Okimoto, S. Koshihara, T. Yamamoto, H. Cui, M. Nomura, Y. Oshima, M. Abdel-Jawad, R. Kato, and R. J. D. Miller, Direct observation of collective modes coupled to molecular orbitaldriven charge transfer, Science 350, 1501 (2015).

[31] B. Köhler, E. Rose, M. Dumm, G. Untereiner, and M. Dressel, Comprehensive transport study of anisotropy and ordering phenomena in quasi-one-dimensional (TMTTF) ${ }_{2} X$ salts $(X=$ $\left.\mathrm{PF}_{6}, \mathrm{AsF}_{6}, \mathrm{SbF}_{6}, \mathrm{BF}_{4}, \mathrm{ClO}_{4}, \mathrm{ReO}_{4}\right)$, Phys. Rev. B 84, 035124 (2011).

[32] M. Dressel, M. Dumm, T. Knoblauch, and M. Masino, Comprehensive optical investigations of charge order in organic chain compounds (TMTTF) $)_{2}$, Crystals 2, 528 (2012). 
[33] P. Monceau, F. Y. Nad, and S. Brazovskii, Ferroelectric MottHubbard Phase of Organic (TMTTF) ${ }_{2} X$ Conductors, Phys. Rev. Lett. 86, 4080 (2001).

[34] H. Seo, C. Hotta, and H. Fukuyama, Toward systematic understanding of diversity of electronic properties in low-dimensional molecular solids, Chem. Rev. 104, 5005 (2004).

[35] S. Ishihara, Electronic ferroelectricity in molecular organic crystals, J. Phys.: Condens. Matter 26, 493201 (2014).

[36] S. Tomić and M. Dressel, Ferroelectricity in molecular solids: A review of electrodynamic properties, Rep. Prog. Phys. 78, 096501 (2015).

[37] R. Rösslhuber, E. Rose, T. Ivek, A. Pustogow, T. Breier, M. Geiger, K. Schrem, G. Untereiner, and M. Dressel, Structural and electronic properties of (TMTTF $)_{2} X$ salts with tetrahedral anions, Crystals 8, 121 (2018).

[38] R. Laversanne, C. Coulon, B. Gallois, J. P. Pouget, and R. Moret, Structural and electrical properties of (TMTTF) ${ }_{2} \mathrm{MF}_{6}$ salts $(\mathrm{M}=\mathrm{P}, \mathrm{As}, \mathrm{Sb})$. Role of the anions, J. Phys. Lett. 45, 393 (1984).

[39] B. Liautard, S. Peytavin, G. Brun, and M. Maurin, Tetramethyltetrathiofulvalene hexafluoroarsenate, $\left(\mathrm{C}_{10} \mathrm{H}_{12} \mathrm{~S}_{4}\right)_{2} \mathrm{AsF}_{6}$, Cryst. Struct. Commun. 11, 1841 (1982).

[40] B. Liautard, S. Peytavin, G. Brun, and M. Maurin, Corrélations structurales dans la série (TMTTF $)_{2}$ X, J. Phys. IV France 43, 1453 (1982).

[41] S. Kitou, T. Fujii, T. Kawamoto, N. Katayama, S. Maki, E. Nishibori, K. Sugimoto, M. Takata, T. Nakamura, and H. Sawa, Successive Dimensional Transition in (TMTTF) ${ }_{2} \mathrm{PF}_{6}$ Revealed by Synchrotron X-ray Diffraction, Phys. Rev. Lett. 119, 065701 (2017).

[42] T. Granier, B. Gallois, L. Ducasse, A. Fritsch, and A. Filhol, $4 \mathrm{~K}$ crystallographic and electronic structures of (TMTTF) $)_{2} \mathrm{X}$ salts $\left(\mathrm{X}^{-}: \mathrm{PF}_{6}^{-}, \mathrm{AsF}_{6}^{-}\right)$, Synth. Met. 24, 343 (1988).

[43] See Supplemental Material at http://link.aps.org/supplemental/ 10.1103/PhysRevResearch.3.L032043 for (1) the experimental details, (2) the systematic shift of the SR peak, (3) the detailed characteristics of the $\mathrm{THz}$ emission, (4) discussions on the length scale of the long-range charge correlations, (5) the estimation of the laser heating, (6) the time evolutions of the photoinduced changes, (7) the photoinduced spectra used in Fig. 5(c), and (8) a discussion on the temperature dependence below $T_{\mathrm{CO}}$.

[44] G. Coslovich, B. Huber, W.-S. Lee, Y. -D. Chuang, Y. Zhu, T. Sasagawa, Z. Hussain, H. A. Bechtel, M. C. Martin, Z. -X. Shen, R. W. Schoenlein, and R. A. Kaindl, Ultrafast charge localization in a stripe-phase nickelate, Nat. Commun. 4, 2643 (2013).

[45] L. DelFreo, A. Painelli, and Z. G. Soos, Giant Infrared Intensity of the Peierls Mode at the Neutral-Ionic Phase Transition, Phys. Rev. Lett. 89, 027402 (2002).

[46] It has been theoretically pointed out that, in the mixed-stack system, the infrared intensity of the dimerization mode divergently increases near the transition point [45]. The absence of such behavior near $T_{\mathrm{CO}}$ (Fig. 2) could be due to the difference in the lattice structure; $\mathrm{TM}_{2} X$ consists of single-component molecular chains with TM dimers, unlike the aforementioned donor-acceptor stack. The observation also suggests that COto-non-CO domain wall excitations, which may be enhanced near $T_{\mathrm{CO}}$, were out of the current $\mathrm{THz}$ range, if any. Although a charged soliton separating antiphase $\mathrm{CO}$ domains may also emerge below $T_{\mathrm{CO}}$ [36], we speculate that it is difficult to identify; since the $\mathrm{CO}$ domains are comparably large to the crystal size [Fig. 3(c)], the solitons might have a small volume fraction, and concomitantly have weak infrared activity to be masked by the intermolecular vibrations.

[47] S. Wall, S. Yang, L. Vidas, M. Chollet, J. M. Glownia, M. Kozina, T. Katayama, T. Henighan, M. Jiang, T. A. Miller, D. A. Reis, L. A. Boatner, O. Delaire, and M. Trigo, Ultrafast disordering of vanadium dimers in photoexcited $\mathrm{VO}_{2}$, Science 362, 572 (2018).

[48] L. Guérin, J. Hébert, M. Buron-LeCointe, S. I. Adachi, S. Y. Koshihara, H. Cailleau, and E. Collet, Capturing OneDimensional Precursors of a Photoinduced Transformation in a Material, Phys. Rev. Lett. 105, 246101 (2010).

[49] R. W. Boyd, Nonlinear Optics, 3rd ed. (Academic, New York, 2008).

[50] H. Itoh, K. Itoh, K. Goto, K. Yamamoto, K. Yakushi, and S. Iwai, Efficient terahertz-wave generation and its ultrafast optical modulation in charge ordered organic ferroelectrics, Appl. Phys. Lett. 104, 173302 (2014).

[51] In a triclinic crystal all of the $\chi^{(2)}$ tensor components are nonzero [49]. Hence the $\mathrm{THz}$ emission along the $b^{\prime}$ axis may occur as observed, although it is perpendicular to the molecular stacks (the $a$ axis) or the expected direction of macroscopic polarization $\boldsymbol{P}$.

[52] M. Sotome, N. Kida, S. Horiuchi, and H. Okamoto, Visualization of ferroelectric domains in a hydrogen-bonded molecular crystal using emission of terahertz radiation, Appl. Phys. Lett. 105, 041101 (2014).

[53] M. Meneghetti, R. Bozio, I. Zanon, C. Pecile, C. Ricotta, and M. Zanetti, Vibrational behavior of molecular constituents of organic superconductors: TMTSF, its radical cation and the sulphur analogs TMTTF and TMTTF $^{+}$, J. Chem. Phys. 80, 6210 (1984).

[54] S. Hirose, A. Kawamoto, N. Matsunaga, K. Nomura, K. Yamamoto, and K. Yakushi, Reexamination of ${ }^{13} \mathrm{C}-\mathrm{NMR}$ in (TMTTF $)_{2} \mathrm{AsF}_{6}$ : Comparison with infrared spectroscopy, Phys. Rev. B 81, 205107 (2010).

[55] M. Dumm, M. Abaker, M. Dressel, and L. K. Montgomery, Charge order in (TMTTF) ${ }_{2} \mathrm{PF}_{6}$ investigated by infrared spectroscopy, J. Low Temp. Phys. 142, 609 (2006).

[56] T. Knoblauch and M. Dressel, Charge disproportionation in (TMTTF $)_{2} X\left(X=\mathrm{PF}_{6}, \mathrm{AsF}_{6}\right.$ and $\left.\mathrm{SbF}_{6}\right)$ investigated by infrared spectroscopy, Phys. Status Solidi C 9, 1158 (2012).

[57] M. Dumm, B. Salameh, M. Abaker, L. K. Montgomery, and M. Dressel, Magnetic and optical studies of spin and charge ordering in (TMTTF) ${ }_{2} \mathrm{AsF}_{6}$, J. Phys. IV France 114, 57 (2004).

[58] R. Świetlik, B. Barszcz, A. Pustogow, and M. Dressel, Raman spectroscopy evidence of domain walls in the organic electronic ferroelectrics (TMTTF $)_{2} X\left(X=\mathrm{SbF}_{6}, \mathrm{AsF}_{6}, \mathrm{PF}_{6}\right)$, Phys. Rev. B 95, 085205 (2017).

[59] While charge disproportionation above $T_{\mathrm{CO}}$ has not been reported in NMR studies [70-72], the electron spin resonance (ESR) study has succeeded in observing a corresponding signal [73]. One reason may be a slow timescale of NMR measurements (typically in the $\mathrm{MHz}$ range) compared to the current $\mathrm{THz}$ measurements or ESR measurements $(\sim 10 \mathrm{GHz})$, which might hinder dynamic charge fluctuations faster than a nanosecond timescale. 
[60] The $\mathrm{THz}$ detector senses a linear superposition of the probe $\mathrm{THz}$ light and the pump-beam-induced $\mathrm{THz}$ emission. In order to filter out the latter contribution from the signal, an incident probe beam was modulated with an optical chopper in both experiments.

[61] T. Yamaguchi, K. Asada, H. Yamakawa, T. Miyamoto, K. Iwano, T. Nakamura, N. Kida, and H. Okamoto, Photoexcitation of a one-dimensional polarization-inverted domain from the charge-ordered ferroelectric ground state of (TMTTF $)_{2} \mathrm{PF}_{6}$, Phys. Rev. B 99, 245104 (2019).

[62] J. T. Kindt and C. A. Schmuttenmaer, Theory for determination of the low-frequency time-dependent response function in liquids using time-resolved terahertz pulse spectroscopy, J. Chem. Phys. 110, 8589 (1999).

[63] G. Coslovich, A. F. Kemper, S. Behl, B. Huber, H. A. Bechtel, T. Sasagawa, M. C. Martin, A. Lanzara, and R. A. Kaindl, Ultrafast dynamics of vibrational symmetry breaking in a charge-ordered nickelate, Sci. Adv. 3, e1600735 (2017).

[64] K. Yamamoto, S. Iwai, S. Boyko, A. Kashiwazaki, F. Hiramatsu, C. Okabe, N. Nishi, and K. Yakushi, Strong optical nonlinearity and its ultrafast response associated with electron ferroelectricity in an organic conductor, J. Phys. Soc. Jpn. 77, 074709 (2008).

[65] H. Itoh, R. Fujiwara, Y. Kawakami, K. Yamamoto, Y. Nakamura, H. Kishida, and S. Iwai, Modulation of terahertz emission in time-domain waveform via a photoinduced phase transition in a charge ordered organic ferroelectric, Appl. Phys. Lett. 112, 093302 (2018).

[66] V. Vescoli, L. Degiorgi, K. P. Starkey, and L. K. Montgomery, Anisotropy in the optical response of (TMTTF $)_{2} \mathrm{X}\left(\mathrm{X}=\mathrm{PF}_{6}\right.$ and $\mathrm{Br}$ ) Bechgaard salts, Solid State Commun. 111, 507 (1999).
[67] M. Först, R. I. Tobey, H. Bromberger, S. B. Wilkins, V. Khanna, A. D. Caviglia, Y.-D. Chuang, W. S. Lee, W. F. Schlotter, J. J. Turner, M. P. Minitti, O. Krupin, Z. J. Xu, J. S. Wen, G. D. Gu, S. S. Dhesi, A. Cavalleri, and J. P. Hill, Melting of Charge Stripes in Vibrationally Driven $\mathrm{La}_{1.875} \mathrm{Ba}_{0.125} \mathrm{CuO}_{4}$ : Assessing the Respective Roles of Electronic and Lattice Order in Frustrated Superconductors, Phys. Rev. Lett. 112, 157002 (2014).

[68] C. Shao, H. Lu, H.-G. Luo, and R. Mondaini, Photoinduced enhancement of bond order in the one-dimensional extended Hubbard model, Phys. Rev. B 100, 041114(R) (2019).

[69] M. Schüler, Y. Murakami, and P. Werner, Nonthermal switching of charge order: Dynamical slowing down and optimal control, Phys. Rev. B 97, 155136 (2018).

[70] D. S. Chow, F. Zamborszky, B. Alavi, D. J. Tantillo, A. Baur, C. A. Merlic, and S. E. Brown, Charge Ordering in the TMTTF Family of Molecular Conductors, Phys. Rev. Lett. 85, 1698 (2000).

[71] F. Zamborszky, W. Yu, W. Raas, S. E. Brown, B. Alavi, C. A. Merlic, A. Baur, S. Lefebvre, and P. Wzietek, Influence of charge order on the ground state of TMTTF conductors, J. Phys. IV France 12, 139 (2002).

[72] S. Fujiyama and T. Nakamura, Redistribution of electronic charges in spin-Peierls state in (TMTTF) $)_{2} \mathrm{AsF}_{6}$ observed by ${ }^{13} \mathrm{C}$ NMR, J. Phys. Soc. Jpn. 75, 014705 (2006).

[73] S. Yasin, B. Salameh, E. Rose, M. Dumm, H.-A. Krug von Nidda, A. Loidl, M. Ozerov, G. Untereiner, L. Montgomery, and M. Dressel, Broken magnetic symmetry due to charge-order ferroelectricity discovered in (TMTTF) ${ }_{2} X$ salts by multifrequency ESR, Phys. Rev. B 85, 144428 (2012). 